توافق قيم الثد عند جبهة الابتلال كدالة لبعض الصفات المائية للتربة

هشام محمود حسن

حسين عبد المجيد جاسم القهوجي

جامعة الموصل / كلية الزراعة والغابات / قسم علوم التربة والمياه

\begin{abstract}
الخلاصة

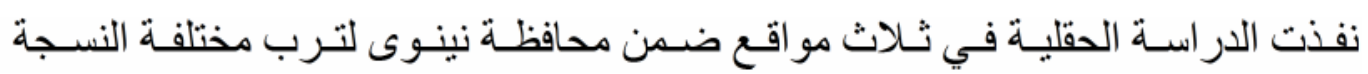

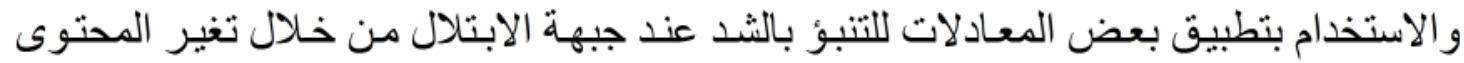

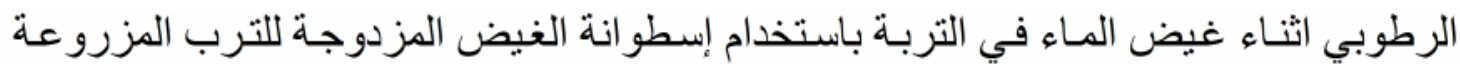

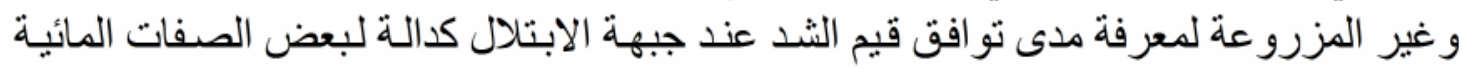

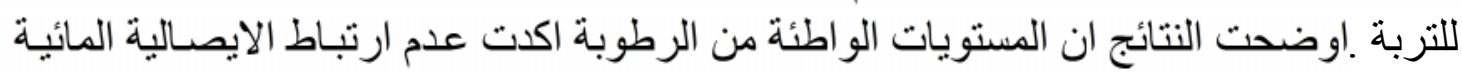

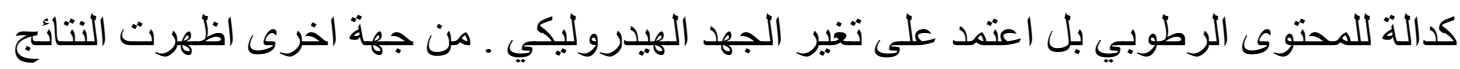

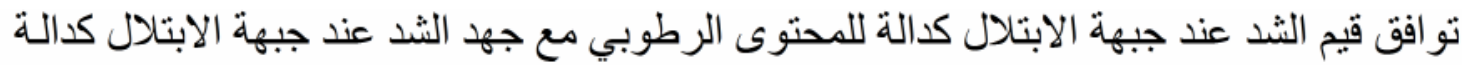
للامتصاصية المحسوبة عند المحتوى الرطوبي الاقل من السعة الحقلية.
\end{abstract}

\title{
Fitting of Matrics Potential at Wetting Front as Function of Some Water Properties
}

\author{
H . A . Jassim \\ H. M. Hassan \\ University of Mosul . College of Agriculture and Forestry Dept of soil \\ and water science
}

\begin{abstract}
This study was conducted in three locations at Ninenva Governorate, with different texure and land use by applying some equation to predict matric potential at wetting front through the variability of moisture contents during the infiltration of water in the soil by using double rings infiltrometer in planted and nonplanted soil to know the correlation of matric potential as function of soil water properties.

Results indicated that the value of hydraulic conductivity as a function of moisture content was not correlated at lower moisture content but related to change of hydraulic head. On the other hand, result revealed that matric potential as a function of both moisture content and
\end{abstract}


calculated sorptivity were best fit at moisture level less than field capacity.

Key words : matrics potential, wetting front, sorptivity, soil water properties. 


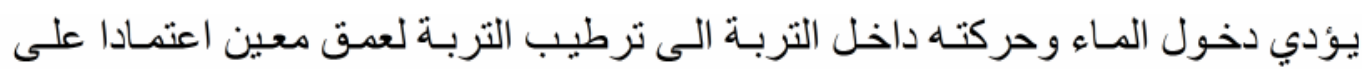

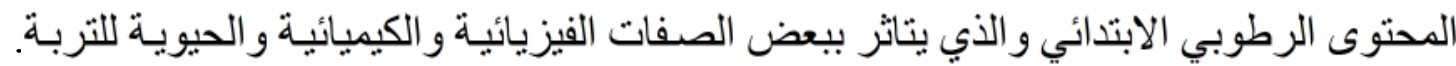

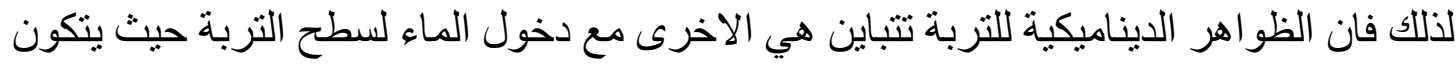

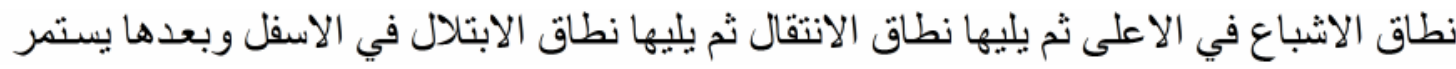

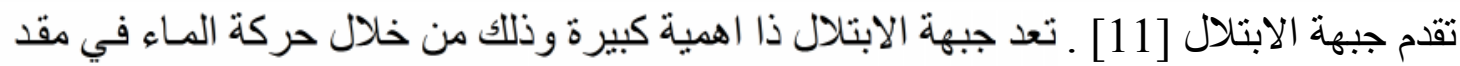

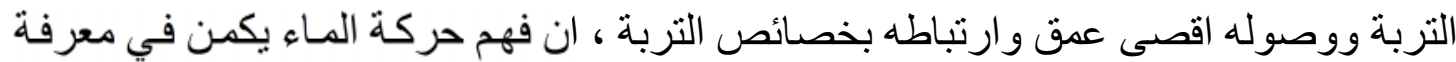

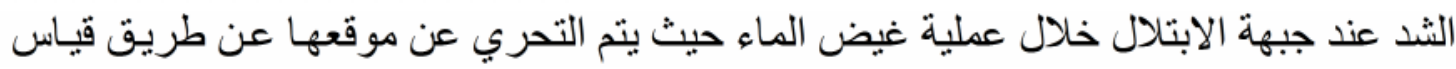

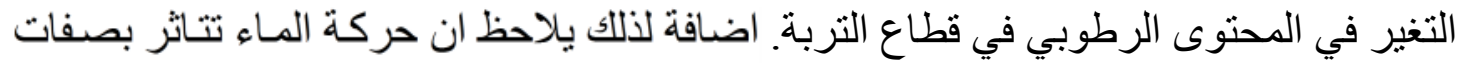

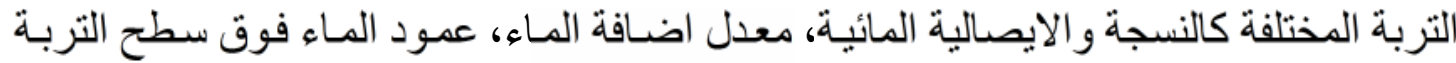

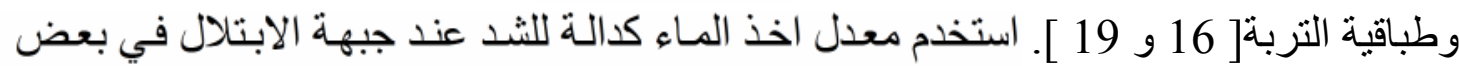

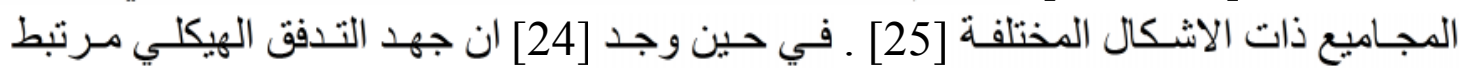

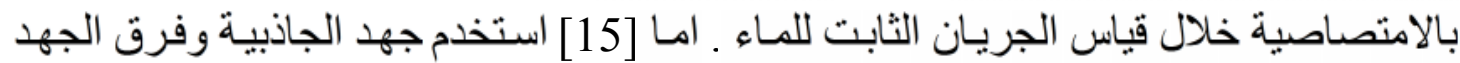

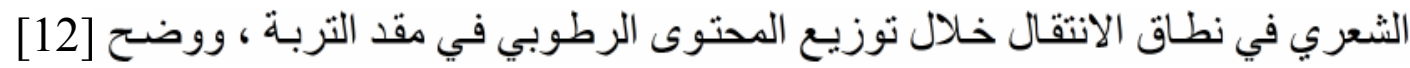

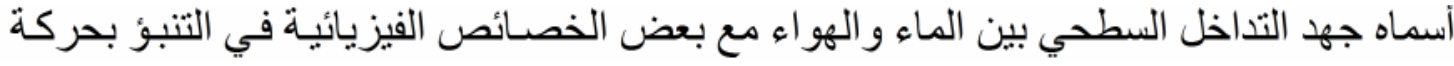

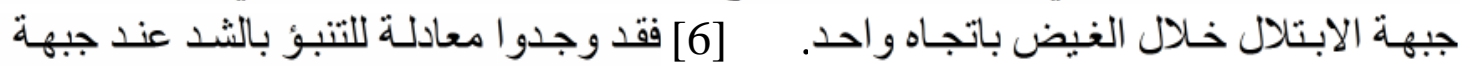

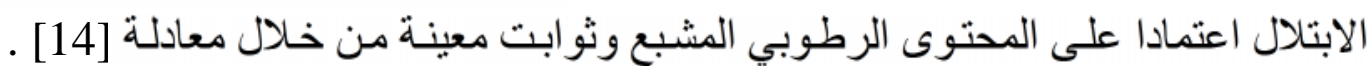

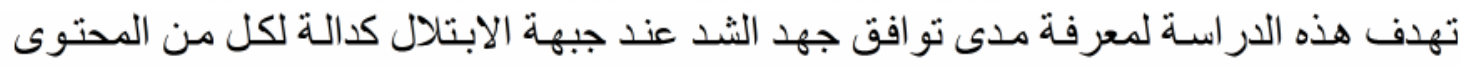
الرطوبي و الامتصاصية المحسوبة لترب مختلفة النسجة و الاستعمال.

\section{مواد وطرق البحث}

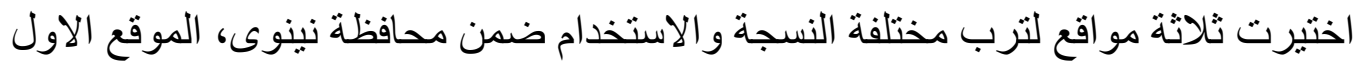

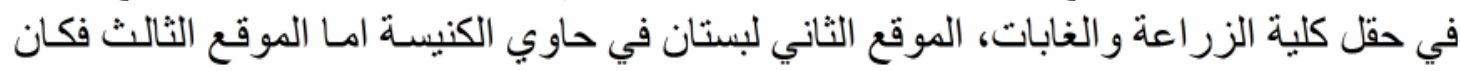

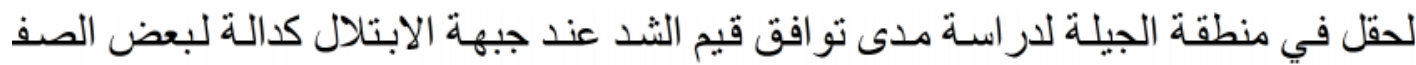

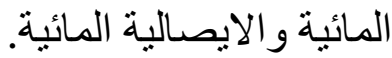

قدر المحتوى الرطوبي بالطريقة الوزنية عن طريق اخذ النماذج بو اسطة مثقاب الريمة في ثناث

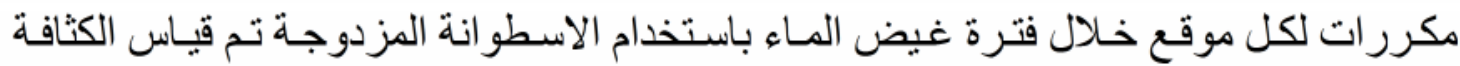

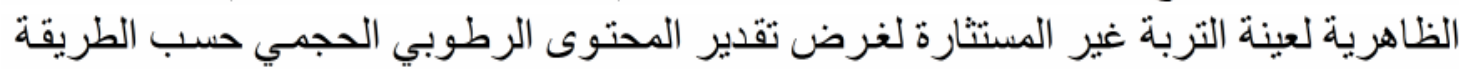

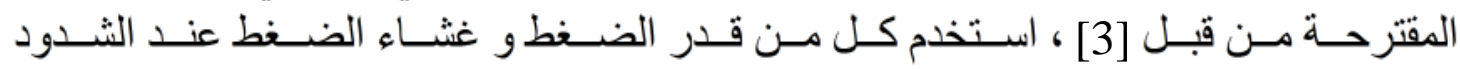

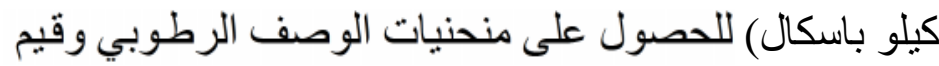

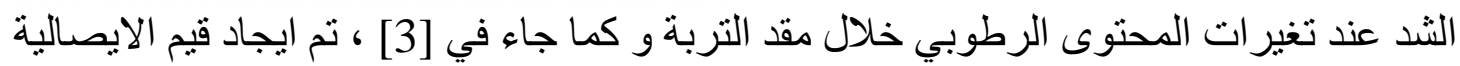

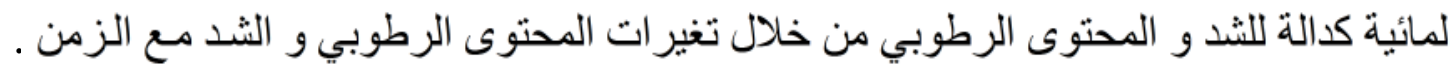

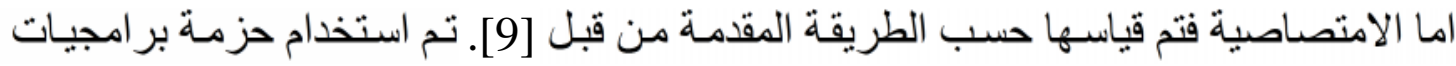
[SPSS Ver.9] المقاسة و المحسوبة بتطبيق معدلات الانحدار المتدرج و البسيط ـ الصفات الفيزيائية و لـئي 
الكيمبائية لترب مواقع الدراسة موضحة في الجدول( ). اعتمد تغيير المحتوى الرطوبي و الثند

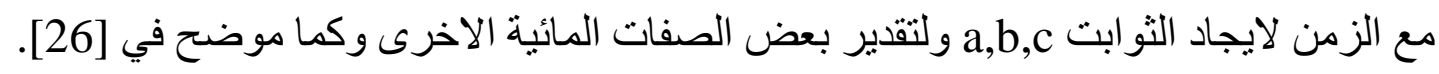
نم ايجاد الثد عند جبهة الابتلال كدالة للمحتوى الرطوبي كما جاء [6] في المعادلة التالية $H_{f}(\theta)=m\left(\frac{\theta_{s}}{a}\right)^{n / 5}\left[\frac{(b-1)}{\left(b+n^{-1}\right)}\right]$

[1]

\section{حيث أن}

الثند $H_{f}(\theta)$

$$
\begin{array}{rr}
(1) \quad \theta_{\mathrm{s}} \\
\mathrm{n}, \mathrm{m}, \mathrm{b}, \mathrm{a}
\end{array}
$$

أما جهد الثد عند جبهة الابتلال كدالة للامتصاصية المحسوبة (Sor) [7] في المعادلتين و على التو الي.

$H_{f}(s o r)=\frac{S^{2}}{-2\left(\theta_{s}-\theta_{i}\right) k_{s}}$

$--[2]$

$$
\begin{aligned}
& \text { حيث أن } \\
& \text { ( ) الثند عند جبهة الابتلال كدالة للامتصاصية } \\
& \text { / }-\theta_{\mathrm{i}} \\
& \text { - S - الامتصاصية سم/ - S } \\
& \text { - الايصالية المائية المشبعة - ks }
\end{aligned}
$$

$S=\left[-2\left(\theta_{s}-\theta_{i}\right) k_{s} m\left(\theta_{s} / a\right)^{m / b}(b-1) /(b+n-1)\right]^{\frac{1}{2}}$

[3]

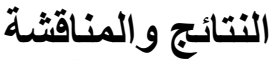

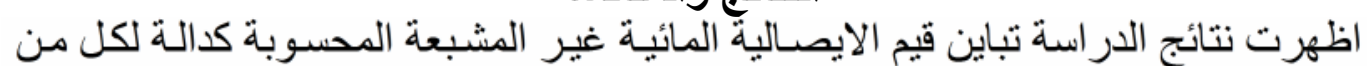

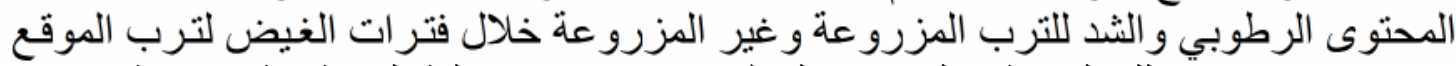

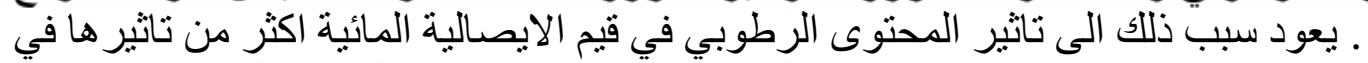

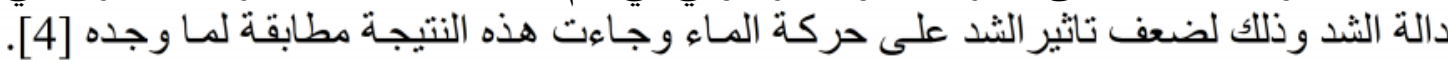

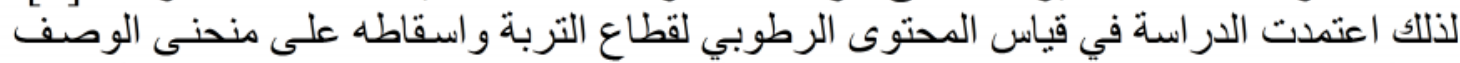

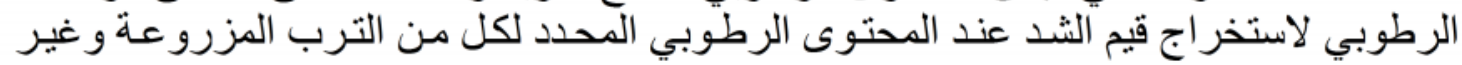




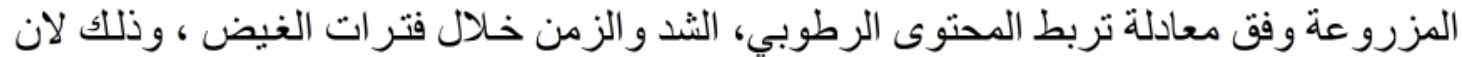

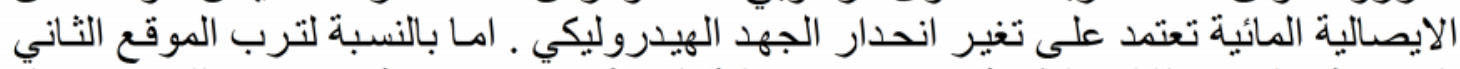

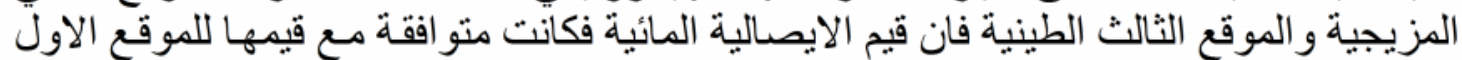

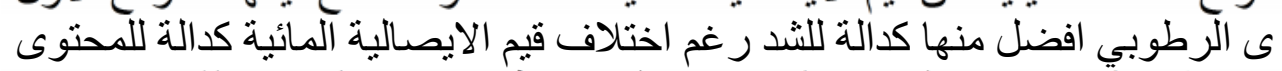

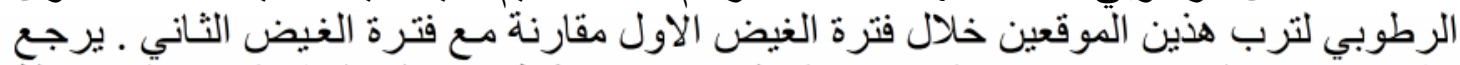

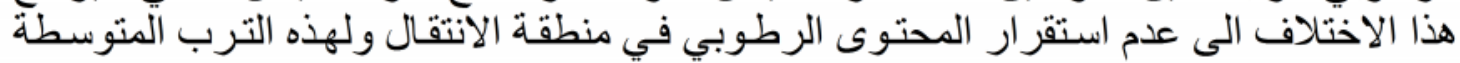

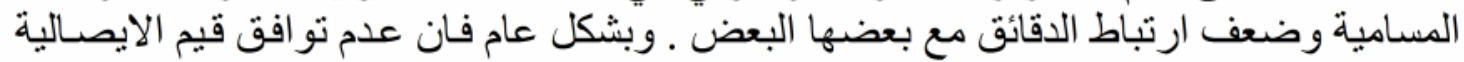

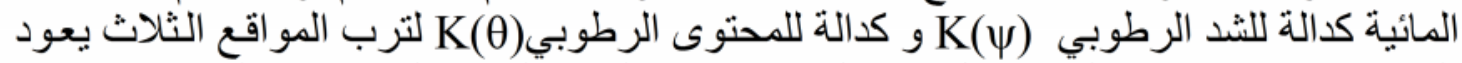

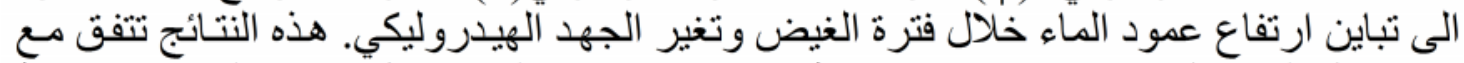

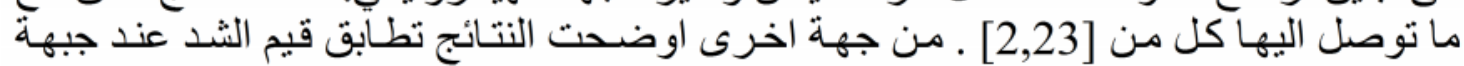

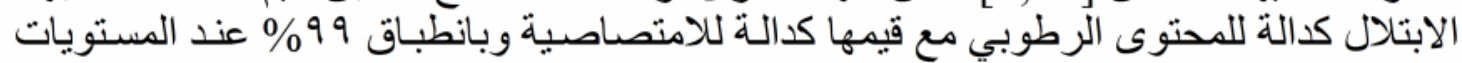

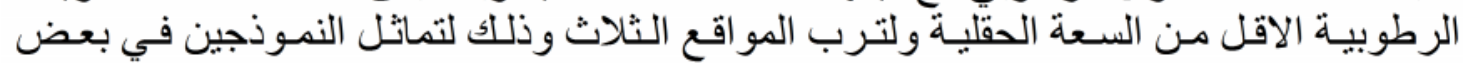

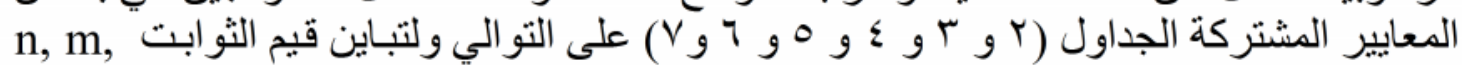

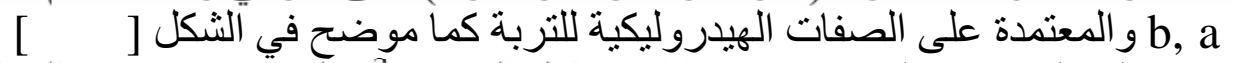

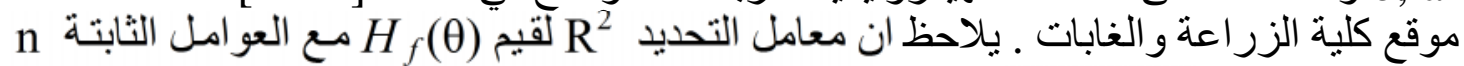

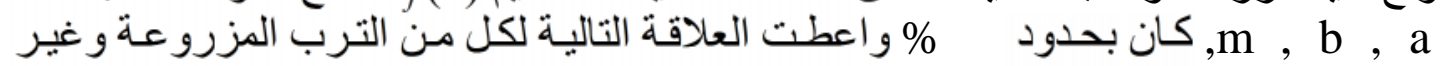
$\mathrm{H}_{\mathrm{f}}[\theta]=252.51-3911.02 \mathrm{~b}-1.90 \mathrm{~m}-1358.1 \mathrm{n}+158.97$

$$
\text { a }
$$
$-----[5]$

$\mathrm{H}_{\mathrm{f}}[\theta]=144.62+1163.90 \mathrm{~b}-19.00 \mathrm{~m}-14.99 \mathrm{n}-131.10 \mathrm{a}$

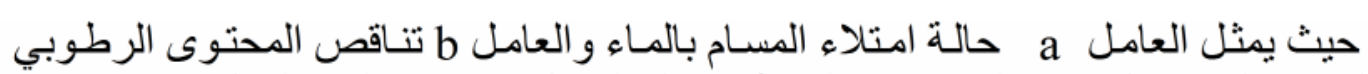

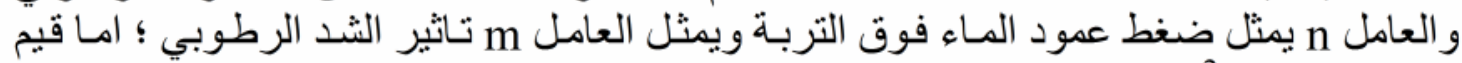

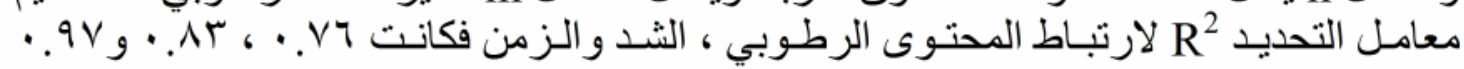

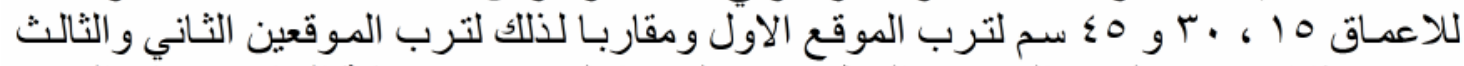

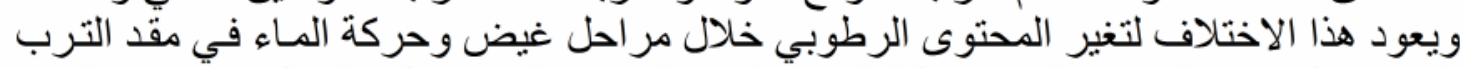

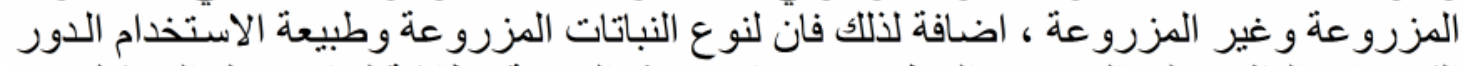

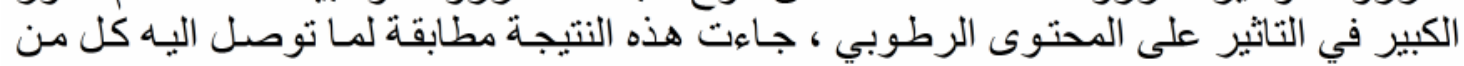
.[22 10626 20

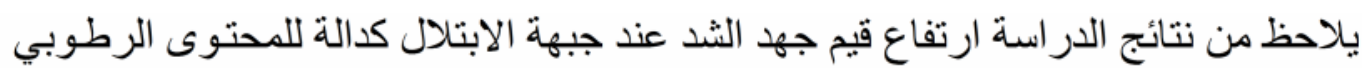

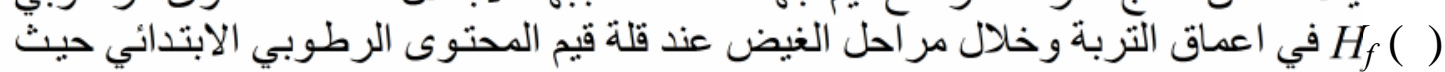

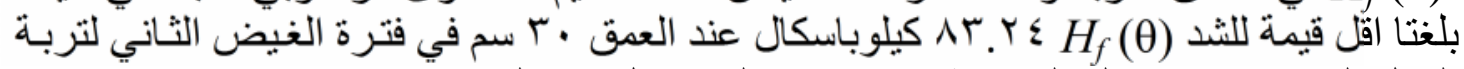

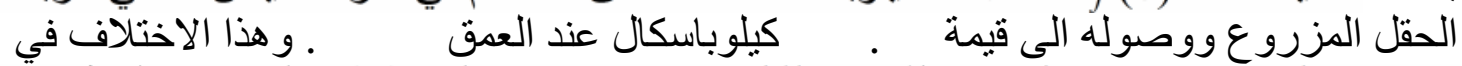

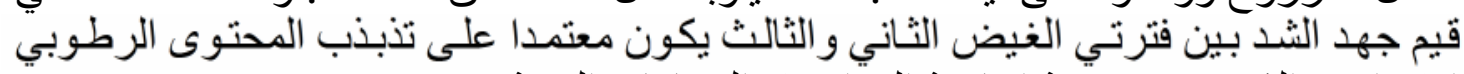
الابتدائي و الذي يعود بدرجة اساسية الى امتلاء المسامات الئينية. 


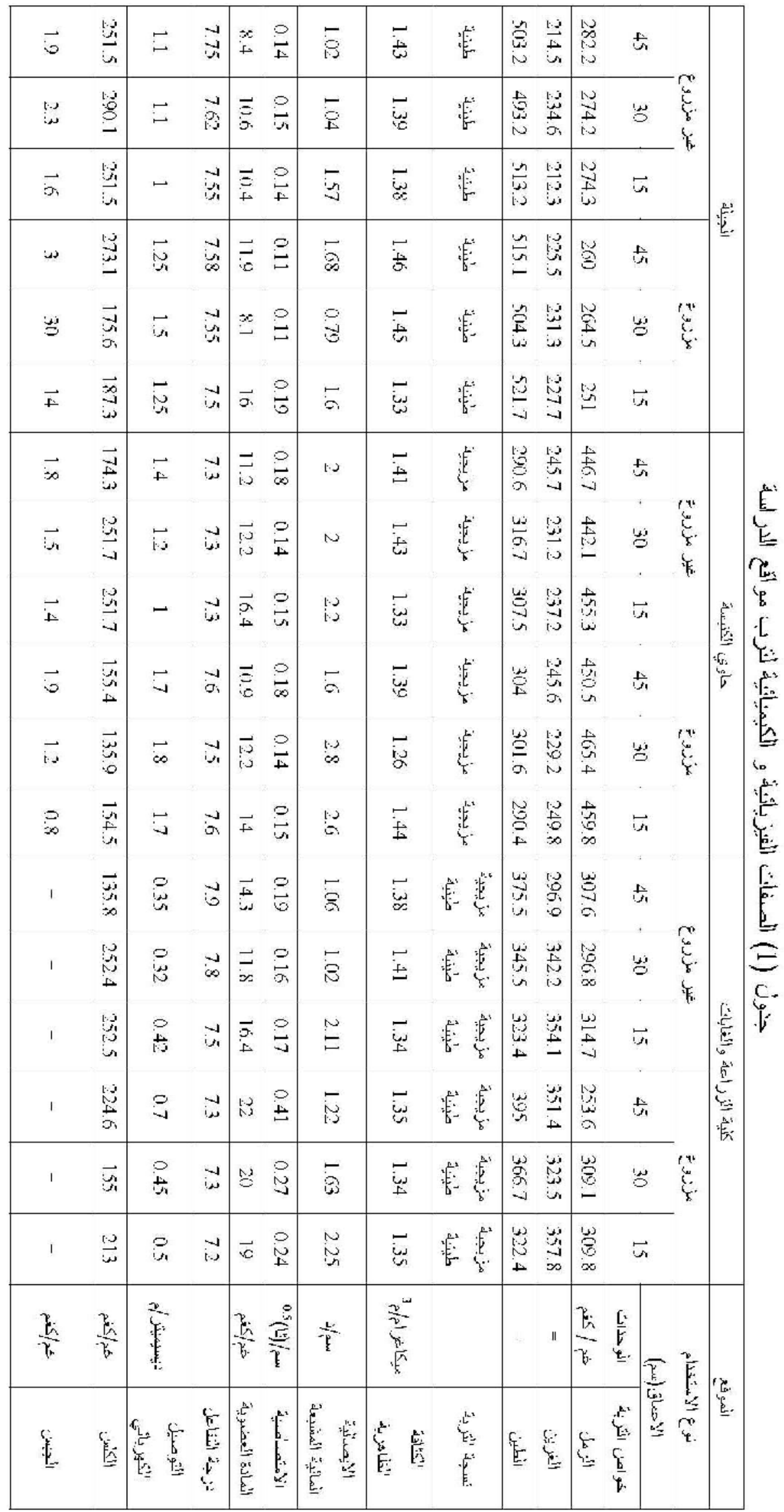




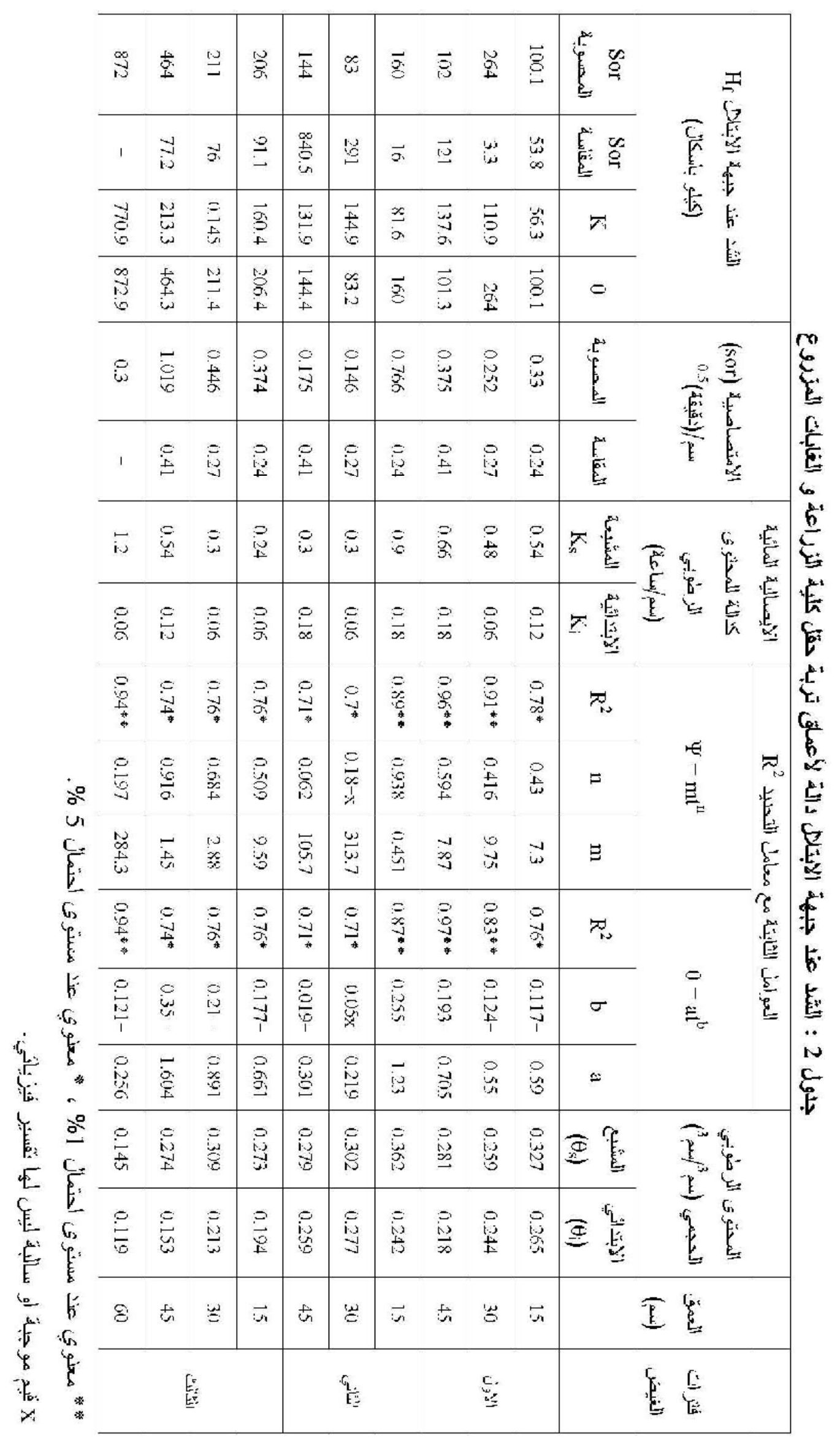




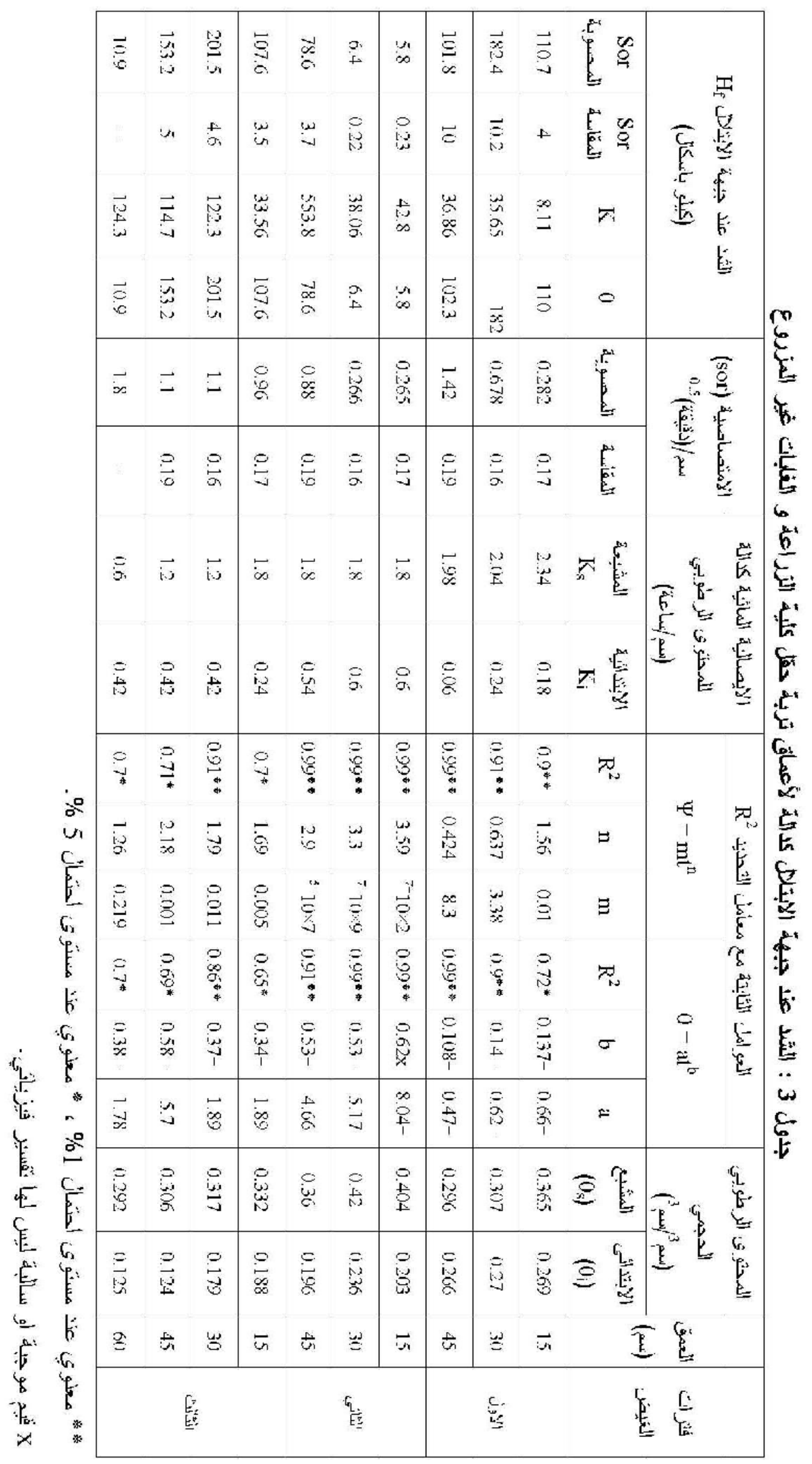




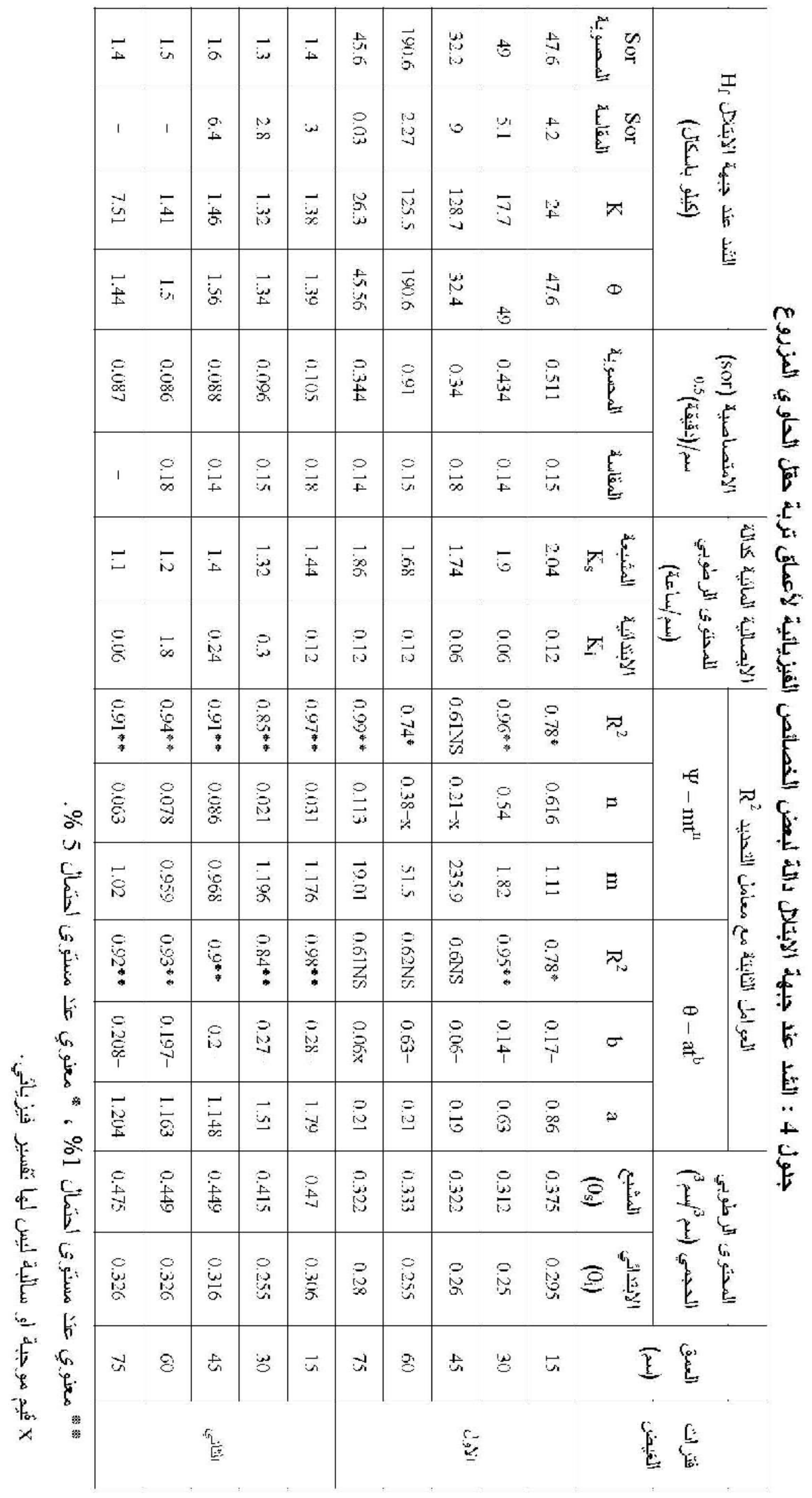




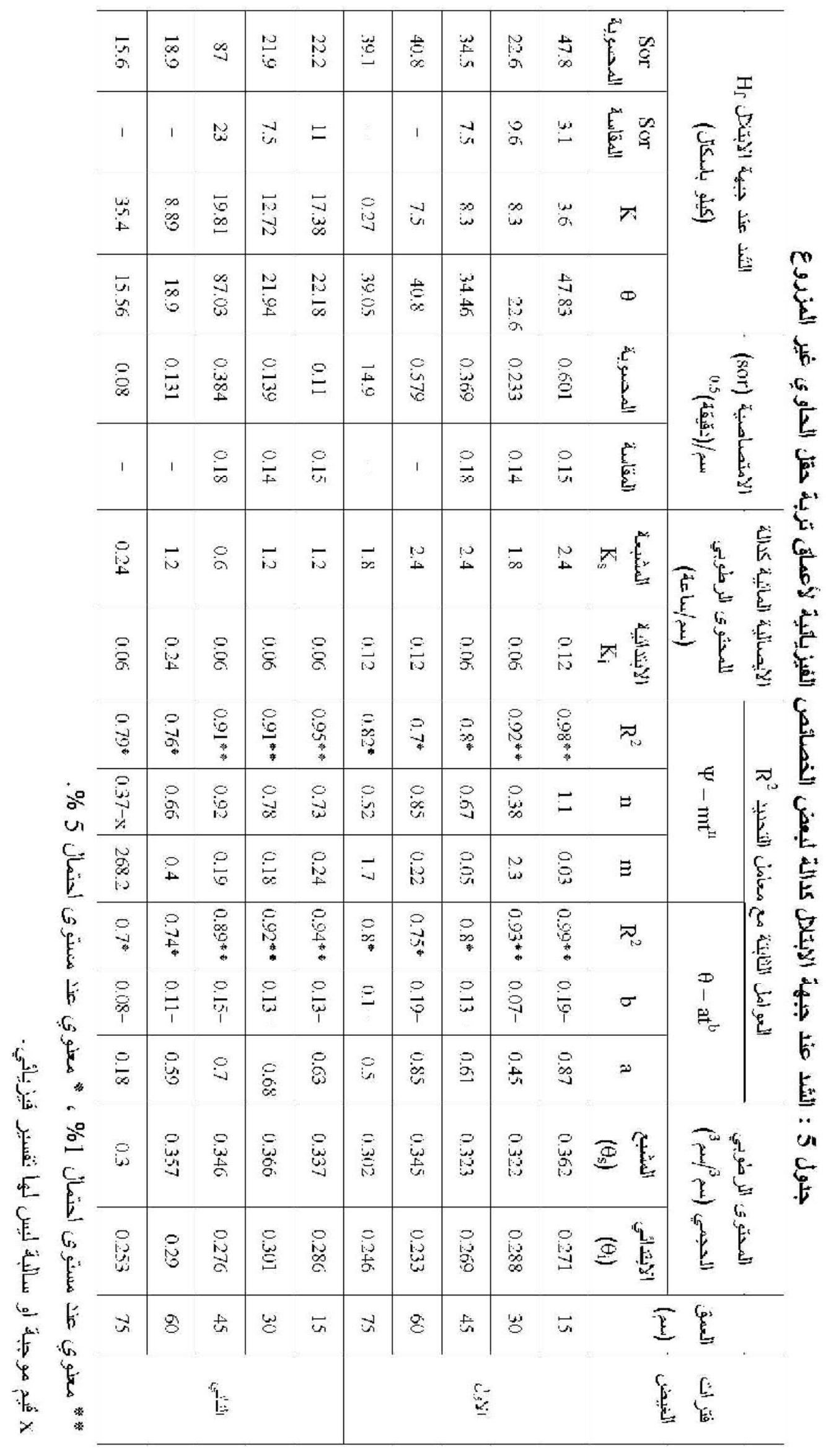




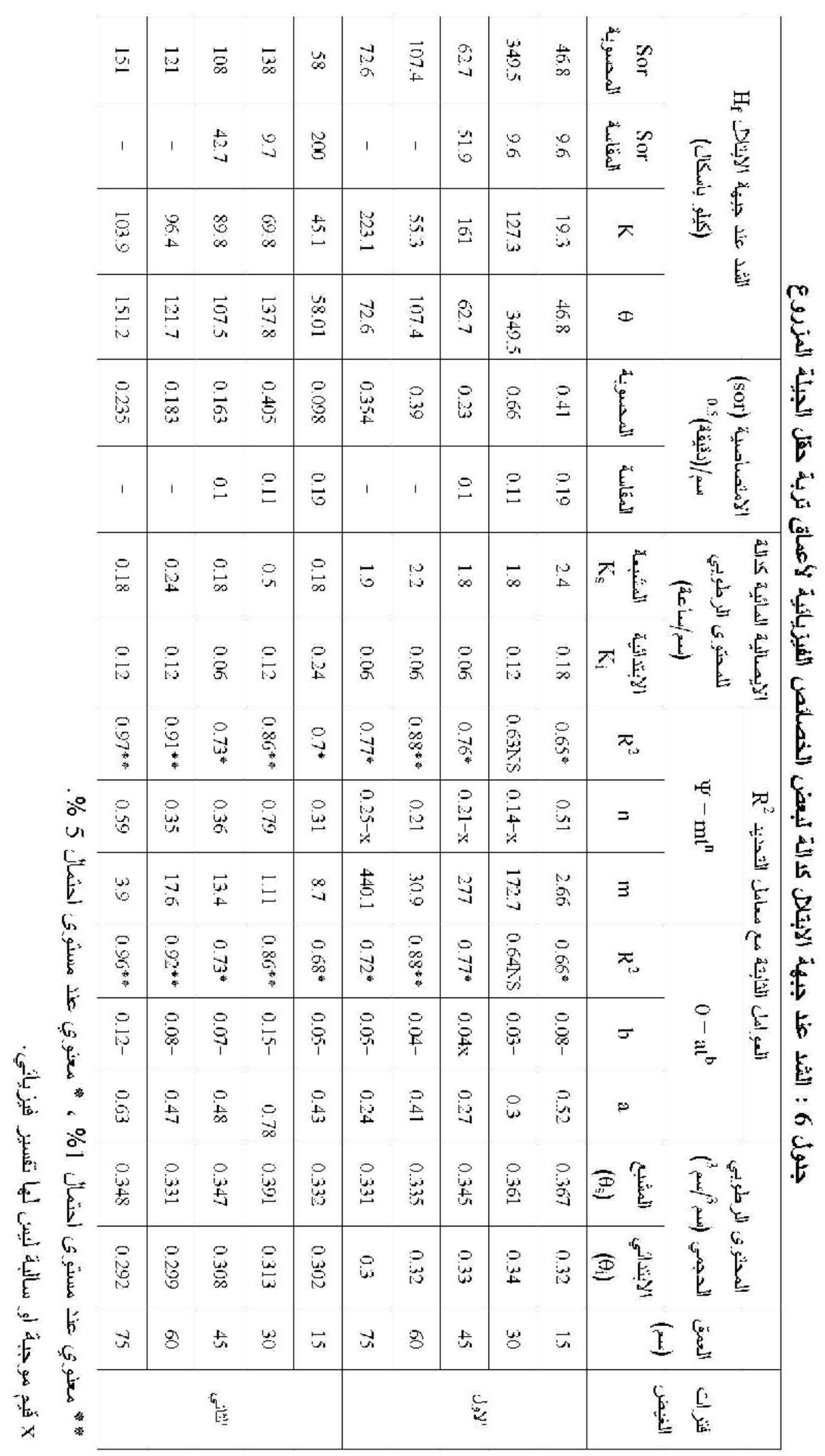




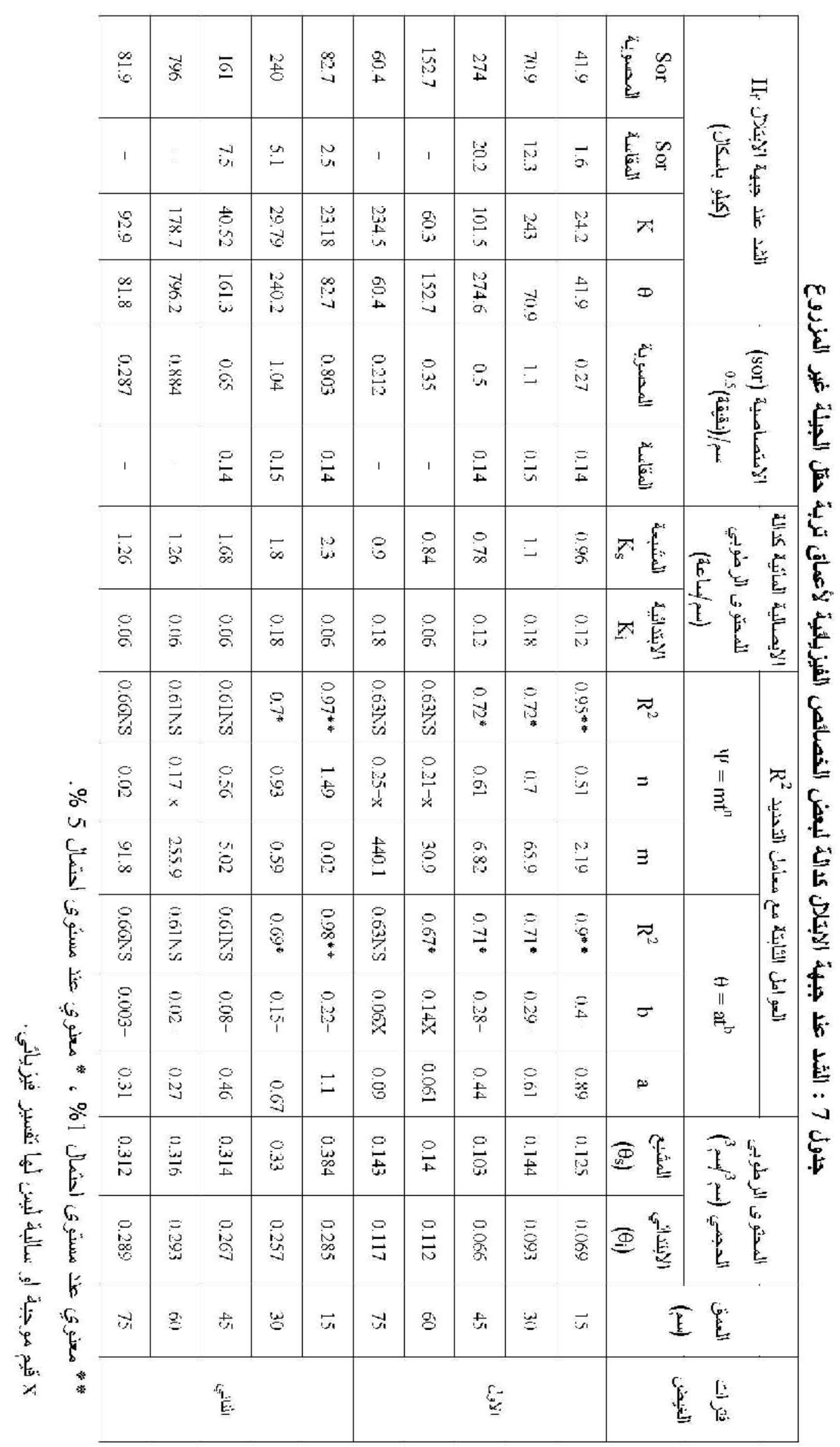



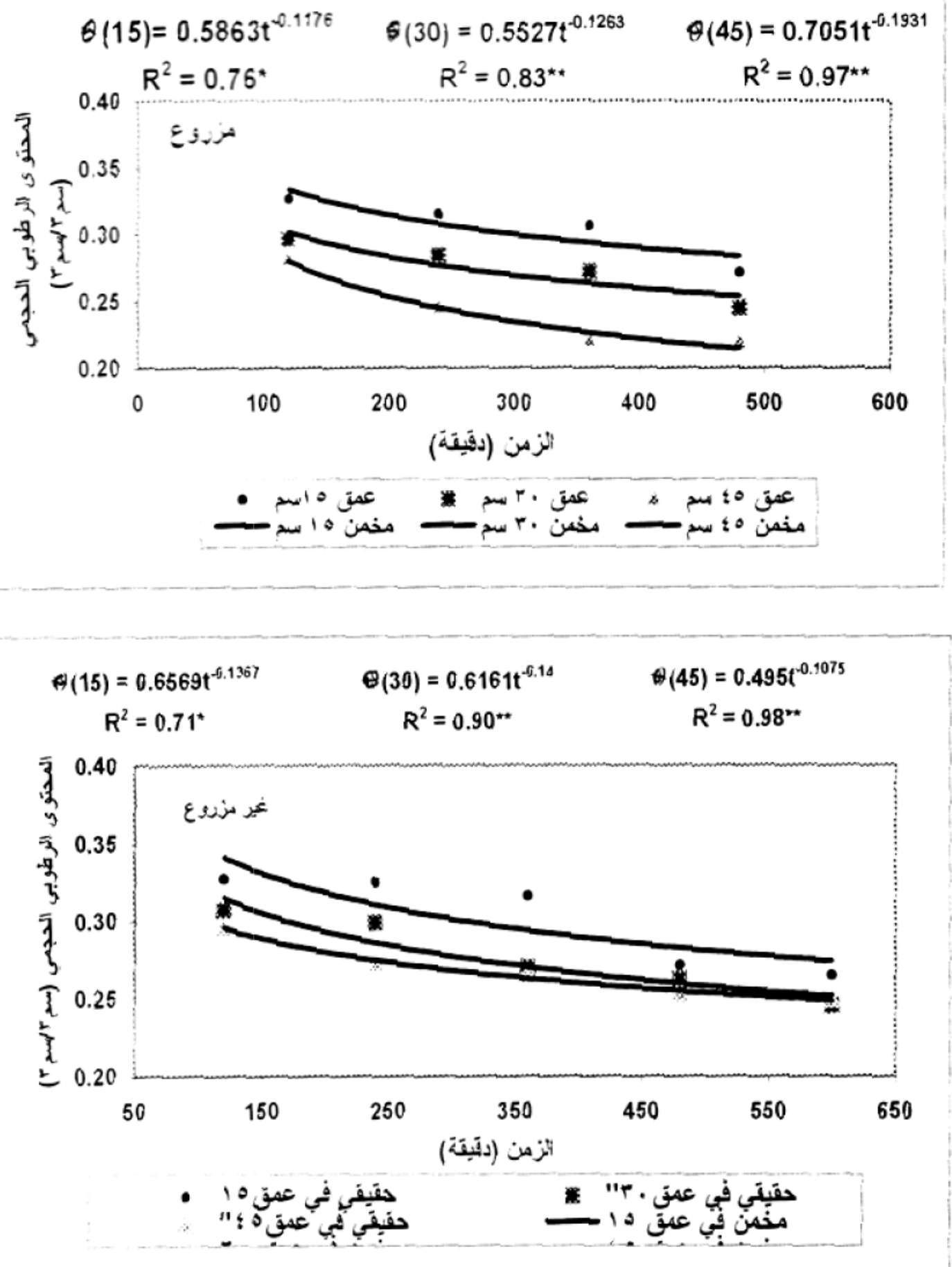

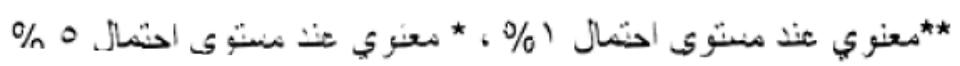


( ) المحتوى الرطوبي لأعماق التربة المختلفة و الزمن خـلال فترة الغيض الأول لحقل كلية الزر اعة والغابات المزروع الزوبع

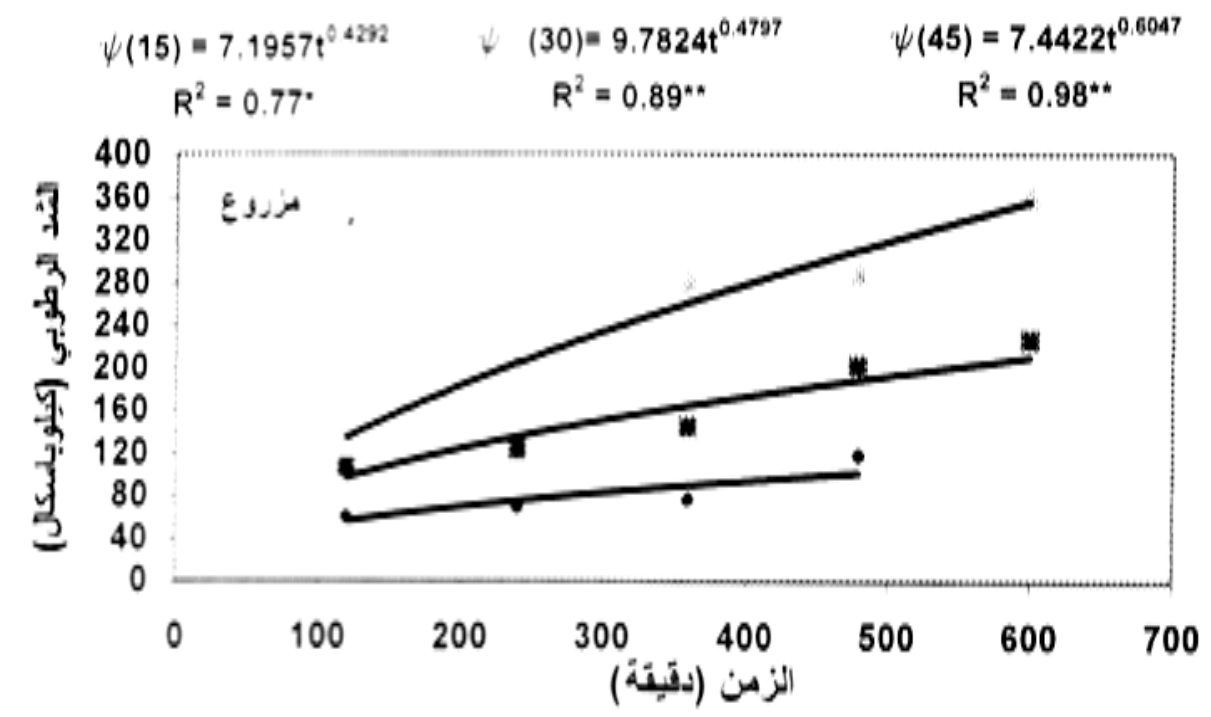

1

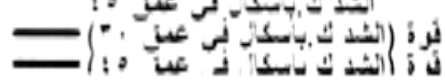

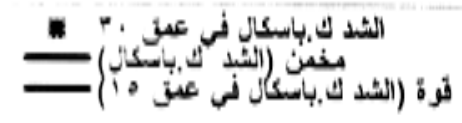
$\psi(15)=0.0053 t^{1.5638}$
$\psi(30)=3.3821 t^{0.6372}$
$\psi(45)=8.3416 t^{0.4246}$
$R^{2}=0.89^{* *}$
$R^{2}=0.91^{\mathrm{nn}}$
$R^{2}=0.96^{n n}$

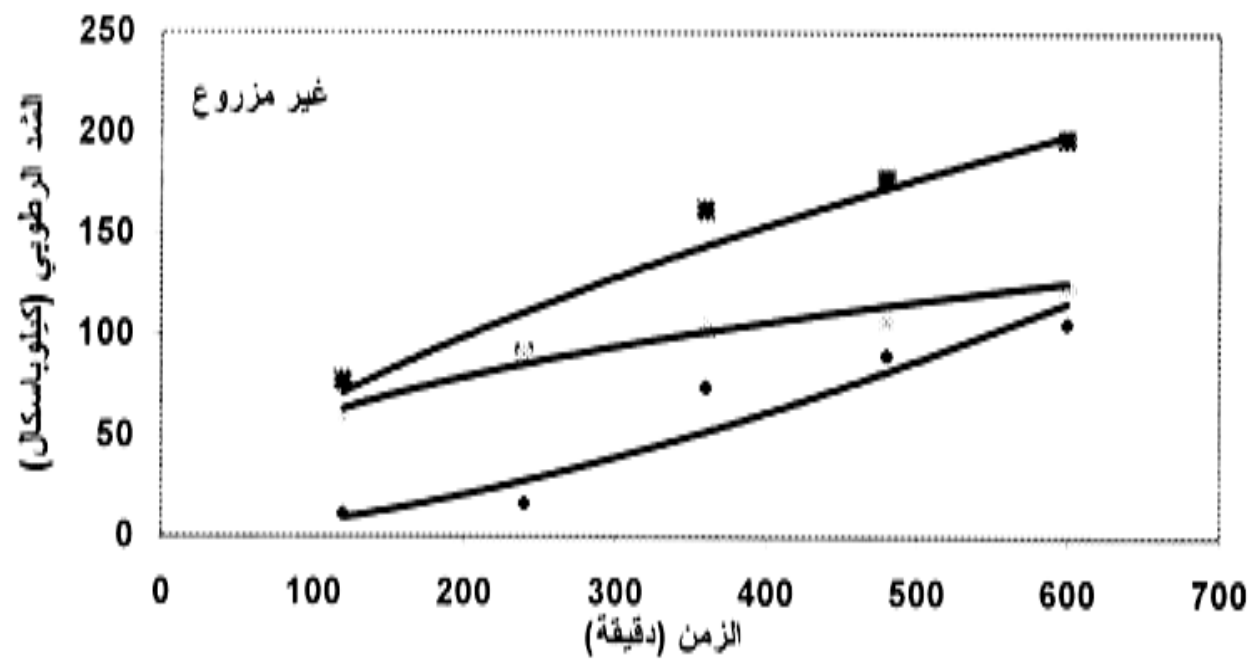

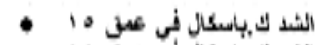

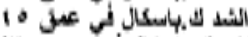

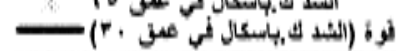

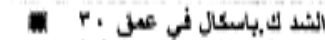

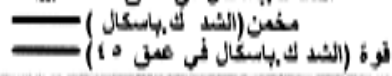

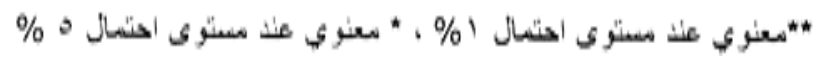


( ) الثد لأعماق التربة المختلفة والزمن خلال فترة الغيض الأول لحقل كلية الزراعة 


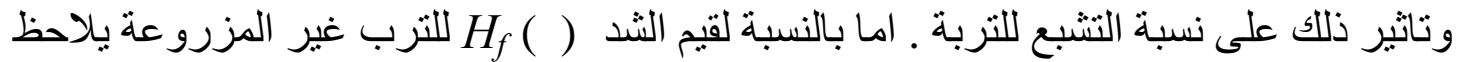

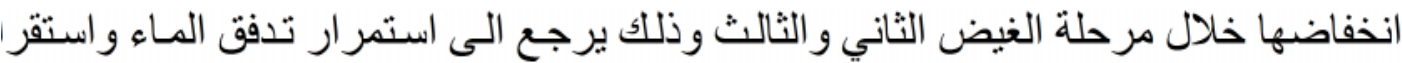

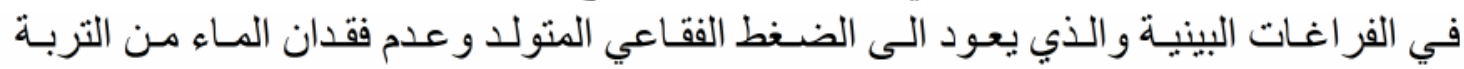

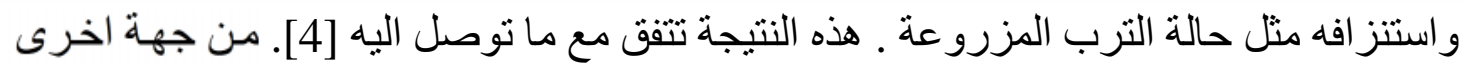

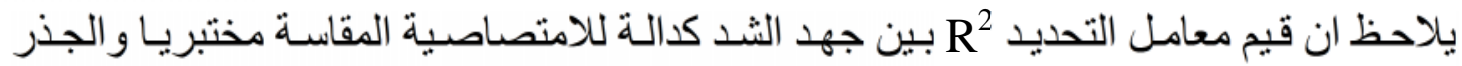

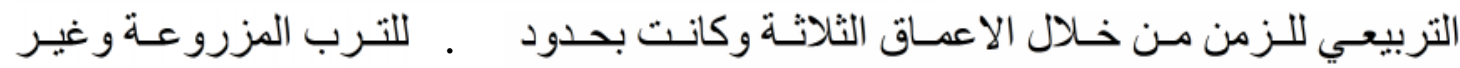

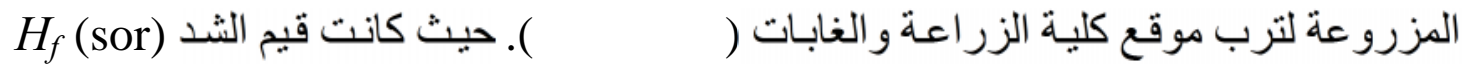

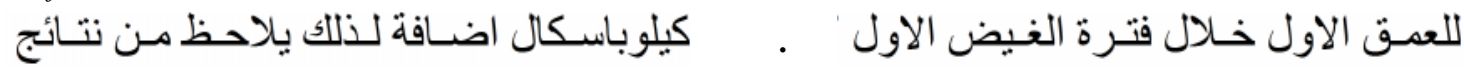

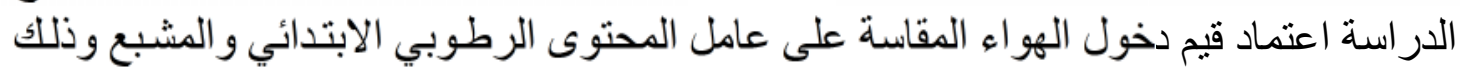

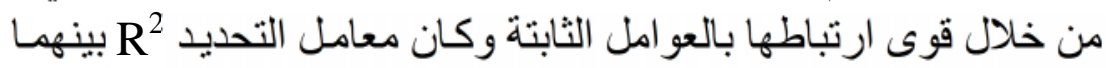

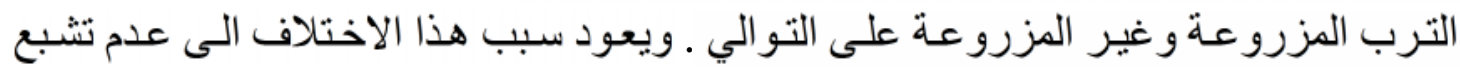

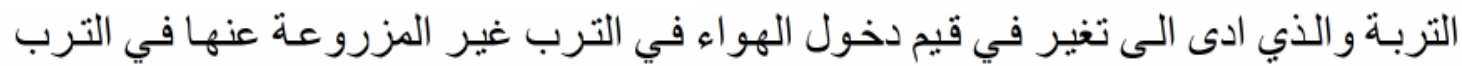

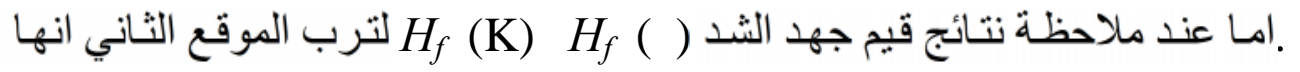

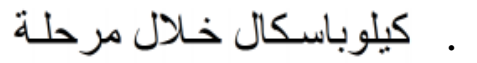

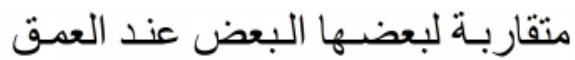

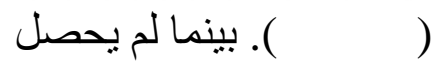
الغيض الثاني واستمر التوافق لغاية العمق التقان

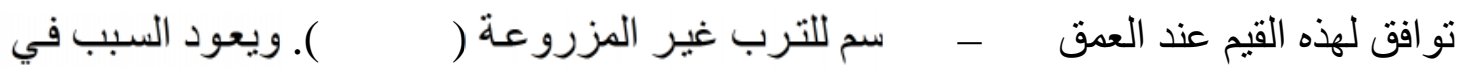

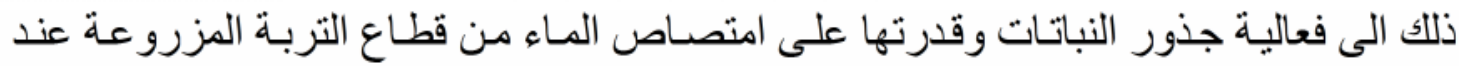

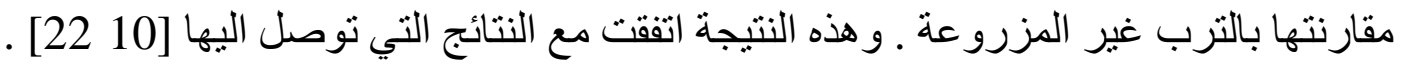

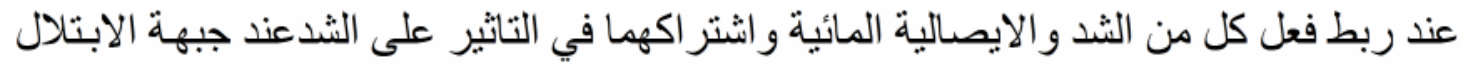
كدالة للايصالية المائية

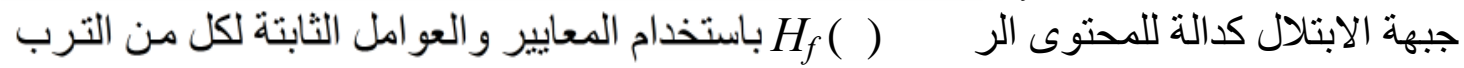

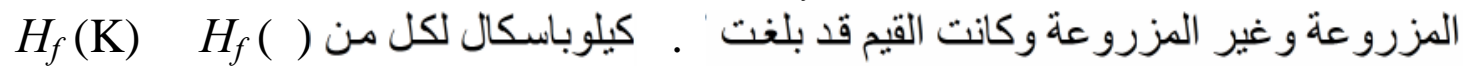

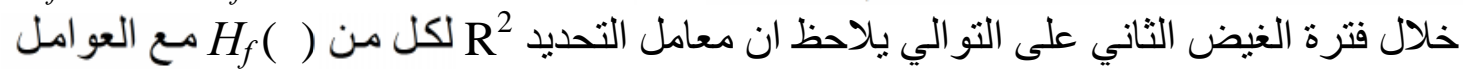

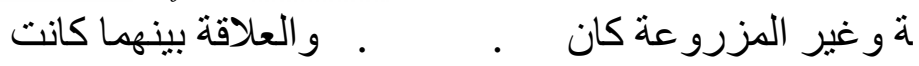

$H_{f}(\theta)=43.26-21.13 \mathrm{a}-73.57 \mathrm{~b}-0.03 \mathrm{~m}+14.34 \mathrm{n}$

$H_{f}(\theta)=28.59-50.64 \mathrm{a}-81.26 \mathrm{~b}+0.37 \mathrm{~m}-23.14 \mathrm{n}$

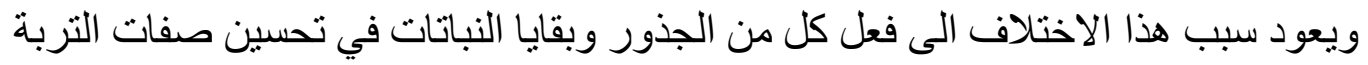

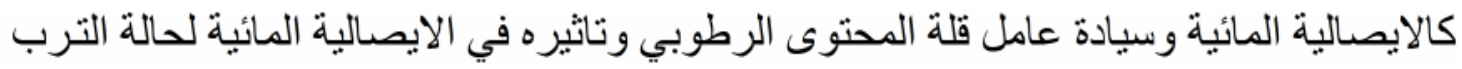

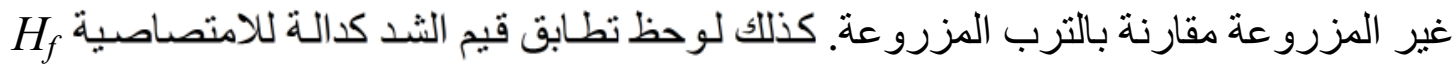
(sor)

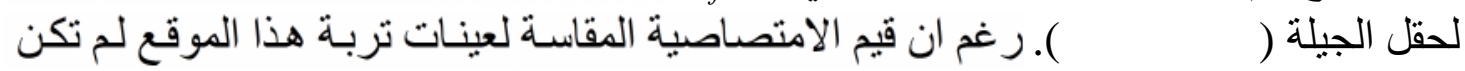

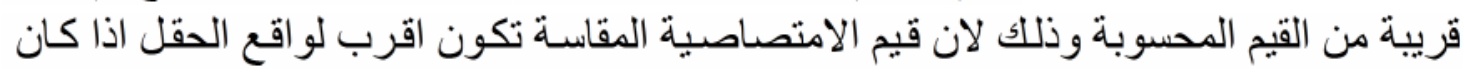

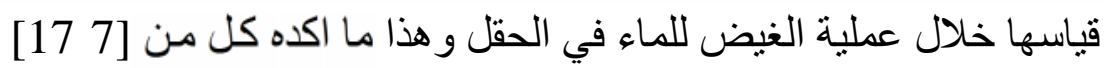

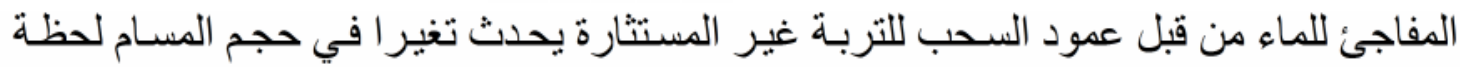


و هذه النتيجة تتفق مع ما توصل اليها كل من [5 5 18 8] ـ ان قيمة معامل

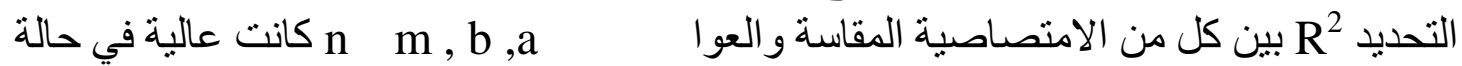

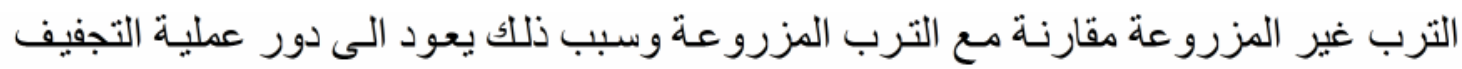

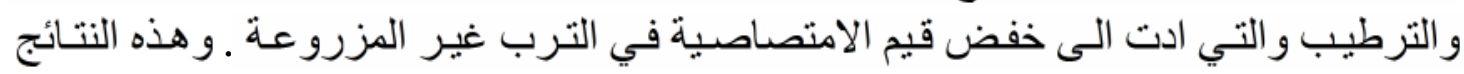

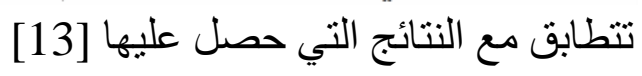

$H_{f}($ sor $)=42.04-127.63 \mathrm{a}+794.9 \mathrm{~b}-0.14 \mathrm{~m}-57.80 \mathrm{n}$

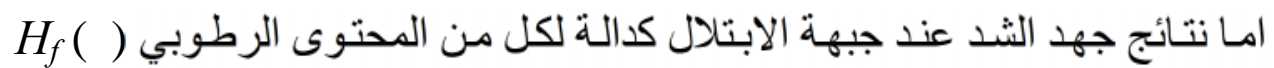

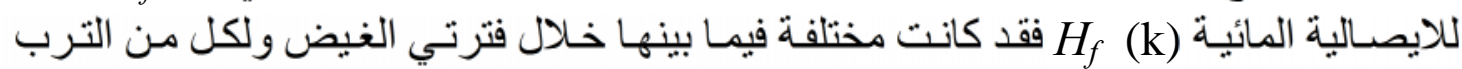

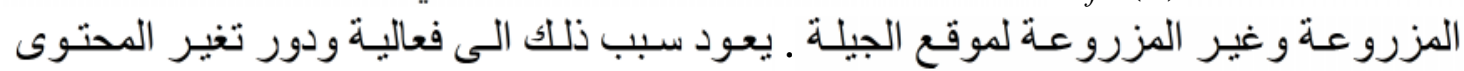

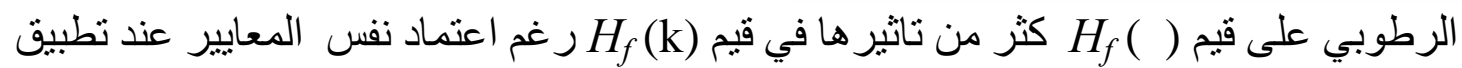
Hf

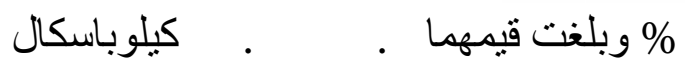

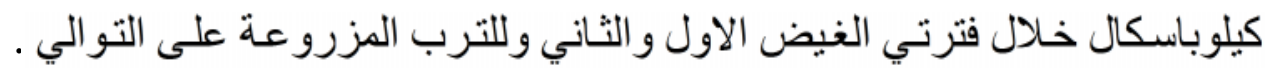

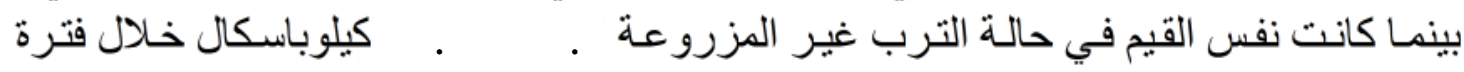

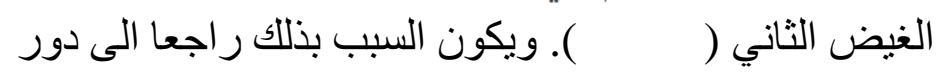
بين كل من

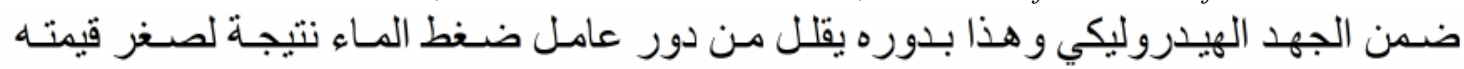

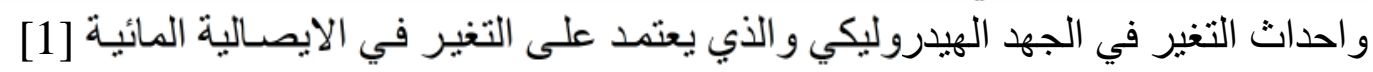

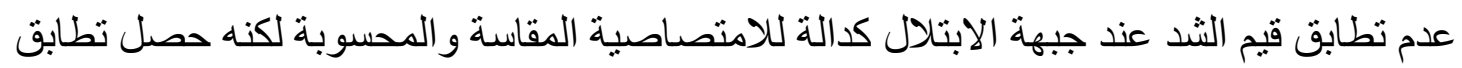

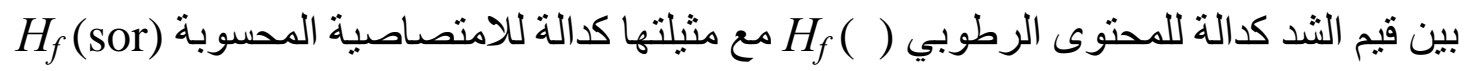

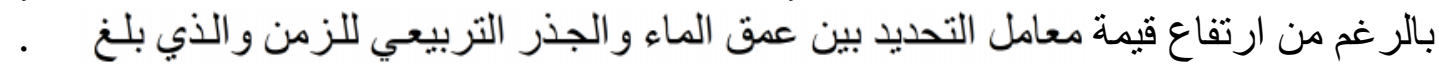

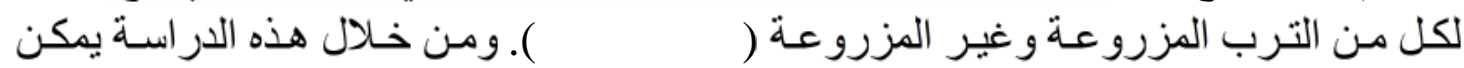

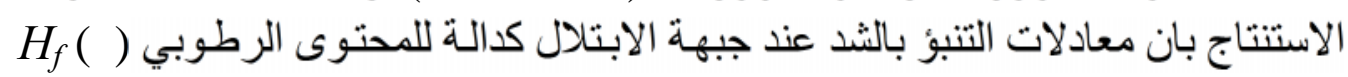

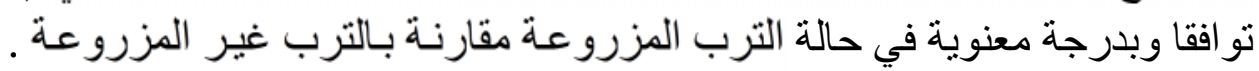

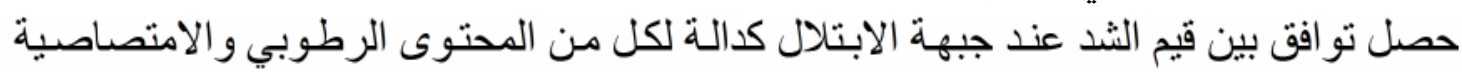

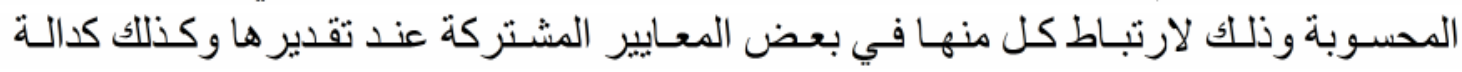

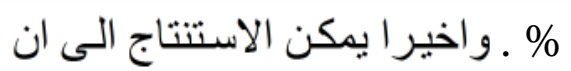

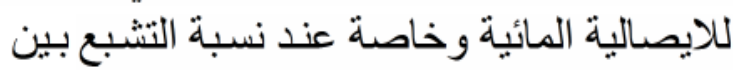

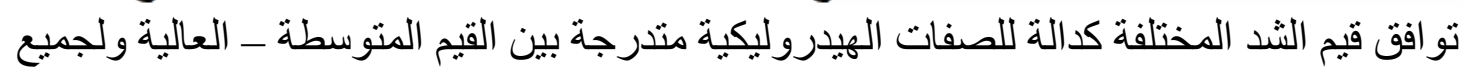

\section{Refrences}

1-Ahuja, L.R, D.L. Nofziger, D. Swartzendruber, and J . D . Ross. Relationship between Green and Ampt parameter based on scalling 
concepts and field - measured hydraulic data . water Resour . Res . 25(7) (1989): $1766-1770$.

2-Ahuja , L .R , R . E . Green, S . K . Chong, and D. R. Nielsen Asimplified functions approach for in situ determination of soil hydraulic conductivities and water characteristics . water Resour Res . 16 (1980): $947-953$.

3-Blake, G.R., and K.H. Hartge. Method of soil analysis. part.1. Physical and Minerlogical Methodes $2^{\text {nd }}$ ed. Mono, Amer. Soc. Agron. Madison, Wisconsin., (1986): 256-266.

4-Braken siek , D . L, and C . A . Onstad . Parameter estimation of the Green and Ampt infiltration equation . Water Resour . Res . 13 (6) (1977): 1009-1012.

5-Chong , S . K ., and R. E . Green . Application of field measured sorptivity for simplified infiltration prediction. P. 88-96 proc. of the symp On hydrologic Transport Modeling . New Orleans . La 10 -11 Dec.ASAE publication (1979) . No - 4-80 .

6-Chong, S . K . R .E . Green, and L . R . Ahuja. Infiltration prediction based on estimation of Green-Ampt wetting front pressure head form measurements of soil water redistribution. Soil Sci. Soc.Am.j. 46 (1982):235-239.

7-Chong, S. K, R. E Green, and L.R. Ahuja. Determination of sorptivity based on in - situ soil water redisribution measurement. Soil Sci. Soc. Am. J. 46 (1982b): 228-230.

8-Clothier, B. E, and K. R. J. Smettem. Combining laboratory and field measuremends to define the hydraulic properties of soil . Soil Sci . Soc . Am . J . 54 (2) (1990): 299-304 .

9-Dirksen, C. Determination of soil water diffusivity by sorptivity measurements. Soil Sci. Soc. Am. Proc. 39 (1975): 22-27.

10-Drost, D. T, and D. Wilcox-Lee. Effect soil matric potential on growth and physiollogical responses of green house grown Asparragus Inter Soc. Horti Sci: 27 (1) (1990) : 467-476.

11-Hansen, V. E. Infiltration and soil water movement during irrigation. Soil Sci. 79 (1955): 93-105. 
12-Kao, C.S, and J.R. Hunt. Prediction of wetting front movement during one-dimensional infiltration into soil water Resour. Res. 32(1) (1996):55-64.

13-Mapa, R. B, R. E. Green, and L. Santo, Temporal variability of soil hydraulic properties with wetting \& drying subsequent to tillage. Soil Sci. Soc. Am. J. 50: (1986) 1133-1138 .

14-Mein, R. G, and C. L. Larson. Modeling infiltration during a steady rain . water Resour Res . 9 (2) (1973) : 384-394.

15-Peck , A. J. Redistribution of soil water after infiltration . Aust. J . Soil Res 9 (1971): 59-71.

16-Philip, J. R. The theory of infiltration 4. Sorptivity and algebraic infiltration equations. Soil Sci. 84 (1957): 257-264.

17-Scotter, D. R, B.E. Clothier, and E. R. Harper. Measuring saturated conductivity and sorptivity using twin ring. Aust . J . Soi / Res . 20 (1982): 295-304 .

18-Sharma, M. L., G. A. Gander, and C. G. Hunt. Spatial variability of infiltration of water shed . J . Hydro . 45 (1980): 101-122 .

19-Skaggs, R. W., D. E. Miller, and R. H. Brooks. Soil water properties . In jensen M . E. (ed). Part 1. Design and operation of farm irrigation system. ASAE Monograph No. 3 (1980): pp $77-123$.

20-Staple , W . J. Infiltration and redistribution of water in vertical columns of loam soil . Soil . Sci . Soc . Am . Proc . 30 (1966): 553558 .

21-Talsma, T.. The effect of initial moisture content and infiltration quantity on redistribution of soil water . Aust . J . Soil . Res . 12 (1974): 15-26.

22-Toyota , K, I . M . Young, and K . Ritz .. Effect of soil matric potential and bulk density an the growth of fusarium oxysporum $\mathrm{F}$. sp. Raphani soil. Biology - Biochemistry. Exeter. 28 (9) (1996): 1139 . 
23-Watson , K .K; and F . D . Whisler. Numerical analysis of drainge of aheterogenous porous medium . Soil Sci . Soc . Am . Proc . 36.(1972) : $251-256$.

24-Wooding, R.A.. Steady infiltration from ashallow circular pond, Water Resour. Res. 4 (1986):1259-1243.

25-Youngs, E. G; P. Bleeds - Harrison, and R. S. Garnett. Water uptake by aggregate. Euro. J. of Soil Sci. 45 (1994): 127-134.

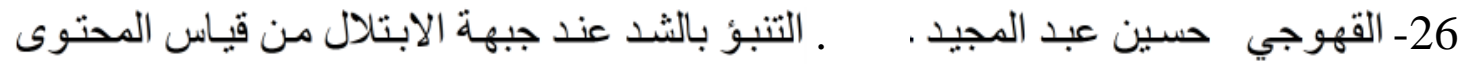

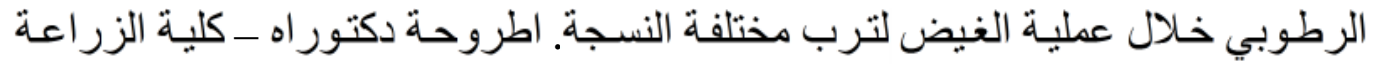

